\title{
AN APPLICATION OF TENSOR ANALYSIS TO THE FIRST VARIATION OF AN INTEGRAL*
}

\author{
BY J. H. TAYLOR
}

The method which J. L. Synge $\dagger$ has used to develop the first and second variations of an integral of a restricted type is readily applicable so far as the first variation is concerned, to a general integral of a regular calculus of variations problem. Let $C$ be a curve in an $n$-dimensional space

$$
x^{\alpha}=x^{\alpha}(u), \quad\left(u_{1} \leqq u \leqq u_{2}\right), \quad(\alpha=1,2, \cdots, n) .
$$

The value of the integral

$$
t=\int_{u_{1}}^{u_{2}} F(x, \dot{x}) d u
$$

will be called the arc length of the curve. $\ddagger$ Let the curve $C$ be imbedded in a one-parameter family of comparison curves

$$
x^{\alpha}=x^{\alpha}(u, v), \quad\left(u_{1} \leqq u \leqq u_{2}, v_{1} \leqq v \leqq v_{2}\right),
$$

such that $C$ is given by $v=0$, that is, $x^{\alpha}(u, 0)=x^{\alpha}(u)$ identically in $u$. We suppose the limits of integration to be unvaried and so the value of the integral $t$ taken along any one of the curves $v=$ constant is a function of $v$ alone. The equations (3) define a surface in terms of the parameters $u$ and $v$. Let the tangent vectors to the $u$ and $v$-curves be designated by $\xi$ and $\eta$ respectively,

* Presented to the Society, December 31, 1926.

$\dagger$ The first and second variations of the length-integral in Riemannian space, Proceedings of the London Mathematical Society, vol. 25 (1925), pp. 247-264.

$\ddagger$ For the conditions imposed on the function $F$ and the notation here employed see the author's paper, Parallelism and transversality in a subspace of a general (Finsler) space, Annals of Mathematics, vol. 28 (1927), pp. $620-628$. 


$$
\xi^{\alpha}=\frac{\partial x^{\alpha}}{\partial u}, \quad \eta^{\alpha}=\frac{\partial x^{\alpha}}{\partial v}
$$

We take as corresponding points on the curve $C$ and its comparison curves the points lying on the curves $u=$ const. The vector $\eta$ will be called the variation or displacement vector. Since the limits of integration are independent of $v$, we have

$$
\frac{\partial t}{\partial v}=\int_{u_{1}}^{u_{2}} \frac{\partial F}{\partial v} \cdot d u
$$

the arguments of $F$ being $x$ and $\xi .^{*}$ On account of the homogeneity property of $F$ we may write $F=F_{\alpha} \xi^{\alpha}$, where $\alpha$ is to be summed from 1 to $n$, and where $F_{\alpha}=\partial F / \partial \dot{x}^{\alpha}$. Hence we have $\partial F / \partial v=\theta F=\theta\left(F_{\alpha} \xi^{\alpha}\right)$, where the differential operator $\theta$ is defined below. Let a symbol $V_{\mu}^{\alpha}(x, \xi)$ be introduced by

$$
V_{\mu}^{\alpha}(x, \xi) \equiv \Gamma_{\mu \beta}^{\alpha} \xi^{\beta}+\frac{1}{2} \frac{\partial \xi^{\gamma}}{\partial u} f_{\mu \beta \gamma} f^{\beta \alpha},
$$

where the arguments of $\Gamma_{\mu \beta}^{\alpha}, f_{\mu \beta \gamma}$ and $f^{\beta \alpha}$ are $x, \xi . \dagger$ Then the $\theta$-process with respect to the curve $C$ when applied to a contravariant vector $X^{\alpha}$ is defined by $\ddagger$

* Any functions employed will be understood to have the arguments $x, \xi$ unless otherwise indicated.

$\dagger$ The quantities in (5) are defined as follows:

$$
f=\frac{1}{2} F^{2}, \quad f_{\alpha \beta}=\frac{\partial^{2} f}{\partial \dot{x}^{\alpha} \partial \dot{x}^{\beta}}, \quad f_{\alpha \beta \gamma}=\frac{\partial f_{\alpha \beta}}{\partial \dot{x}^{\gamma}},
$$

$f^{\beta \alpha}$ is the element of the reciprocal matrix of $f_{\lambda \mu}$ corresponding to the term $f_{\alpha \beta} ; \Gamma_{\mu}^{\alpha}=f^{\alpha \sigma}[\mu \beta, \sigma]$ where

$$
[\mu \beta, \sigma]=\frac{1}{2}\left(\frac{\partial f_{\mu \sigma}}{\partial x^{\beta}}+\frac{\partial f_{\beta \sigma}}{\partial x^{\mu}}-\frac{\partial f_{\mu \beta}}{\partial x^{\sigma}}\right) .
$$

$\ddagger$ Further properties of the operator $\theta$ and the resulting theory of parallelism cannot be given here in detail. Reference may be made to J. H. Taylor, A generalization of Levi-Civita's parallelism and the Frenet formulas, Transactions of this Society, vol. 27 (1925), pp. 252-259. This differentiation process was developed about the same time by J. L. Synge, A generalization of the Riemannian line-element, Transactions of this Society, vol. 27 (1925), pp. 61-67. The $\theta$-process and resulting parallelism are distinct from the corresponding processes as developed by E. Noether, Invarianten beliebiger Differentialausdrïcke, Göttinger Nachrichten, 1918, 


$$
\theta X^{\alpha} \equiv \frac{d X^{\alpha}}{d u}+V_{\beta}^{\alpha}(x, \xi) X^{\beta} .
$$

We adopt the notation of Synge in this connection and designate $\theta X$ with respect to a $v$-curve by $X$ and with respect to a $u$-curve by $\bar{X}$, where $X$ is any tensor.

With this notation, we have

If we assume that

$$
\frac{\partial F}{\partial v}=\hat{F}=\hat{F}_{\alpha} \xi^{\alpha}+F_{\alpha} \hat{\xi}^{\alpha}
$$

$$
\frac{\partial^{2} x^{\alpha}}{\partial u \partial v}=\frac{\partial^{2} x^{\alpha}}{\partial v \partial u}
$$

it follows that $\xi^{\alpha}-\bar{\eta}^{\alpha}=V_{\lambda}^{\alpha}(x, \eta) \xi^{\lambda}-V_{\lambda}^{\alpha}(x, \xi) \eta^{\lambda}$. By means of this relation one obtains

$$
\frac{\partial F}{\partial v}=F_{\alpha} \bar{\eta}^{\alpha}+Q
$$

where $Q$ is an abbreviation for the expression

$$
\hat{F}_{\alpha} \xi^{\alpha}+F_{\alpha}\left(V_{\lambda}^{\alpha}(x, \eta) \xi^{\lambda}-V_{\lambda}^{\alpha}(x, \xi) \eta^{\lambda}\right) .
$$

On expanding these terms and making use of the homogeneity condition, it results that $Q$ is identically zero. Hence, we have

$$
\delta t=\delta v \int_{u_{1}}^{u_{2}} F_{\alpha} \bar{\eta}^{\alpha} d u
$$

where the arguments of $F$ are $x, \xi$ of the curve $C$ defined by (3) for $v=0$, and where the directional derivative $\bar{\eta}^{\alpha}=\theta \eta^{\alpha}$ of the variation vector $\eta$ is with respect to the curve $C$.

From (8) we see that the first variation certainly vanishes if $\bar{\eta}^{\alpha}=0$ along the curve. However, if this condition holds

pp. 37-44, and L. Berwald, Untersuchung der Krïmmung allgemeiner metrischer Räume auf Grund des in ihnen herrschenden Parallelismus, Mathematische Zeitschrift, vol. 25 (1926), pp. 40-73, except when the $\theta$-process is carried out with respect to a curve which satisfies the equations

of Berwald, p. 44.

$$
x^{\prime \prime i}=-\phi^{i}\left(x, x^{\prime}\right)
$$


at every point of $C$, the original and varied curve cannot have a point in common unless they coincide. For $\bar{\eta}^{\alpha}=0$ is the condition for parallel displacement, and under such a displacement the length of a vector when measured with respect to the tangent vector to the curve, as is the case here, remains constant.*

By the homogeneity condition, $\delta t$ may be written in the form

$$
\delta t=\delta v \int_{u_{1}}^{u_{2}} \frac{f_{\alpha \beta} \xi \bar{\eta}^{\beta}}{F} d u .
$$

We now change the form of this expression by means of the relation

$$
\frac{\partial}{\partial u}\left[f_{\alpha \beta} \xi^{\alpha} \eta^{\beta}\right]=\theta\left[f_{\alpha \beta} \xi^{\alpha} \eta^{\beta}\right]=f_{\alpha \beta} \bar{\xi}^{\alpha} \eta^{\beta}+f_{\alpha \beta} \xi^{\alpha} \bar{\eta}^{\beta},
$$

and the choice of a parameter such that $F=1$ along the curve $C$. Then an integration gives

or

$$
\delta t=\delta v\left\{\left.f_{\alpha \beta} \xi \xi^{\beta} \eta^{\beta}\right|_{u_{1}} ^{u_{2}}-\int_{u_{1}}^{u_{2}} f_{\alpha \beta} \bar{\xi} \eta^{\beta} d u\right\}
$$

(9) $\delta t=\delta v\left\{\left.|\eta|_{\xi} \cos (\xi, \eta)_{\xi}\right|_{u_{1}} ^{u_{2}}-\int_{u_{1}}^{u_{2}}|\bar{\xi}|_{\xi}|\eta|_{\xi} \cos (\bar{\xi}, \eta)_{\xi} d u\right\}$,

where $|\eta|_{\xi}$ denotes the length of the vector $\eta$, and $\cos (\xi, \eta)_{\xi}$ is the cosine of the angle between $\xi$ and $\eta$ measured with respect to the tangent vector $\xi$. Here $\bar{\xi}^{\alpha}$ is the principal normal of the curve $C$ and $|\bar{\xi}|_{\xi}$ is the first curvature of the curve. $\dagger$ A curve whose first curvature vanishes will be called a geodesic. From (9) we have the following well known result.

THEOREM 1. A necessary and sufficient condition that the first variation of the length integral of a curve $C$ shall be zero for arbitrary variations except that at the end points the varia-

* J. H. Taylor, Transactions of this Society, loc. cit., p. 259.

$\dagger$ J. H. Taylor, Transactions of this Society, loc. cit., pp. 261, 263. 
tions shall vanish or be orthogonal* (with respect to the line elements of $C$ ) to the curve, is that $C$ shall be a geodesic. The equations of a geodesic referred to the arc length $t$ as parameter are $\dagger$

$$
\bar{\xi}^{\alpha} \equiv \frac{d^{2} x^{\alpha}}{d t^{2}}+\Gamma_{\lambda \mu}^{\alpha}\left(x, \frac{d x}{d t}\right) \frac{d x^{\lambda}}{d t} \frac{d x^{\mu}}{d t}=0, \quad(\alpha=1,2, \cdots, n) .
$$

The quantity $\delta v|\eta|_{\xi} \cos (\bar{\xi}, \eta)_{\xi}$ represents the projection or component of the displacement vector $\eta$ on the principal normal $\bar{\xi}$ of the curve $C$. Hence for variations which cause the integrated term of (9) to vanish at the limits of integration, the first variation of the arc length of a curve depends only on the displacement of the curve in the direction of its principal normal. $\ddagger$

Let $R$ be a region of the surface (3) in which the curves $v=$ constant constitute a one-parameter family of geodesics of the $n$-space. We suppose that through each point of $R$ there passes one, and only one, of the geodesics of the family. Furthermore, let each of the geodesics of the family be cut transversally (orthogonally with respect to the line elements of the geodesics) by a curve $u=u_{1}$. Now if $u=u_{2}$ is another curve in $R$ also cutting the geodesics transversally we see from (9) that the arc length of these geodesics between these two transversal curves is a constant. Conversely, let a given distance be measured off on each geodesic from its point of intersection with the transversal $u=u_{1}$. Then for each point determined in this fashion we have the condition

* The orthogonality here used includes transversality as a special case. See L. Berwald, loc. cit., p. 56.

† These equations in this form were explicitly obtained by Synge, Berwald and the author at about the same time. J. L. Synge, Transactions of this Society, loc. cit., L. Berwald, Über Parallelubertragung in Räumen mit allgemeiner Maszbestimmung, Jahresbereicht der Vereinigung, 1925, p. 217. J. H. Taylor, Reduction of Euler's equations to a canonical form, this Bulletin, vol. 31 (1925), p. 262. One observes that this method of treating the first variation leads directly to the Euler equations in normal form.

$\ddagger$ See W. Blaschke, Vorlesungen über Differentialgeometrie, vol. I, 1921, p. 35 , where this result is given for a curve in euclidean 3-space. 


$$
\left.|\eta| \xi \cos (\xi, \eta)_{\xi}\right|^{u_{2}}=0 \text {. }
$$

By hypothesis $|\eta|_{\xi} \neq 0$, since the region $R$ is simply covered by the geodesics of the family, and hence $\left.\cos (\xi, \eta)_{\xi}\right|_{=0} ^{u_{2}}$, that is, $\eta$ is orthogonal to the geodesic, the angle being measured with respect to the tangent vector to the geodesic. Thus we have a theorem which is a simple extension of Kneser's transversality theorem (Bolza, Vorlesungen uber Variationsrechnung, 1909, p. 339).

THEOREM 2. Let a one-parameter family of geodesics of an $n$-space $(n>2)$ simply cover a region $R$ of a two-dimensional space, and let $C_{1}$ be a curve which cuts each of these geodesics transversally (orthogonally with respect to the tangent vector of the geodesic). Then a necessary and sufficient condition that the arc length in the sense of (2) measured along these geodesics from the curve $C_{1}$ to a second curve $C_{2}$ shall be a constant is that $C_{2}$ shall also cut the geodesics of the family transversally.

Let $K$ be a sub-space of $k$ dimensions $(k<n)$. A vector $\xi$ at a point of $K$ will be said to be orthogonal to $K$ if it is orthogonal to every vector in $K$ at that point. A necessary and sufficient condition for this is that $\xi$ shall be orthogonal to $k$ linearly independent vectors in $K$ at that point, say the tangent vectors to the parametric curves. Hence if we consider the first variation of an integral with respect to a class of curves joining a fixed point with a point in a given $K$-space we have the following result.

TheOREm 3. A necessary and sufficient condition that the first variation of an integral taken along a curve $C$ in an $n$ space, one end point of which is fixed while the other end point is required to lie in a given $k$-dimensional space $K,(k<n)$, shall vanish is that the curve $C$ shall be a geodesic and that it meet the space $K$ orthogonally with respect to the tangent vector of $C$.

The University of Wisconsin 